\title{
BMJ Open Perceptions and experiences of healthcare providers during COVID-19 pandemic in Karachi, Pakistan: an exploratory qualitative study
}

\author{
Anam Shahil Feroz (i) ,1,2 Nousheen Akber Pradhan, ${ }^{2}$ Zarak Hussain Ahmed, ${ }^{2}$ \\ Mashal Murad Shah, ${ }^{2}$ Nargis Asad, ${ }^{3}$ Sarah Saleem, ${ }^{2}$ Sameen Siddiqi (1) ${ }^{2}$
}

To cite: Shahil Feroz A, Pradhan NA, Hussain Ahmed Z, et al. Perceptions and experiences of healthcare providers during COVID-19 pandemic in Karachi, Pakistan: an exploratory qualitative study. BMJ Open 2021;11:e048984. doi:10.1136/ bmjopen-2021-048984

- Prepublication history and additional online supplemental material for this paper are available online. To view these files, please visit the journal online (http://dx.doi.org/10. 1136/bmjopen-2021-048984).

Received 28 January 2021 Accepted 19 July 2021

Check for updates

(C) Author(s) (or their employer(s)) 2021. Re-use permitted under CC BY-NC. No commercial re-use. See rights and permissions. Published by BMJ.

1Dalla Lana School of Public Health, Institute of Health Policy Management and Evaluation, University of Toronto, Toronto, Ontario, Canada

${ }^{2}$ Department of Community Health Sciences, Aga Khan University, Karachi, Sindh, Pakistan

${ }^{3}$ Department of Psychiatry, Aga Khan University, Karachi, Sindh, Pakistan

Correspondence to

Dr Anam Shahil Feroz;

anam.shahil@mail.utoronto.ca

\section{ABSTRACT}

Objective To explore healthcare provider's perspectives and experiences of the barriers and facilitators to treat and manage COVID-19 cases.

Design and setting We conducted an exploratory qualitative study using a purposive sampling approach, at a private tertiary care teaching hospital in Karachi, Pakistan. Study data were analysed manually using the conventional content analysis technique.

Participants Key-informant interviews (KIIs) were conducted with senior management and hospital leadership and in-depth interviews (IDIs) were conducted with front-line healthcare providers.

Results A total of 31 interviews (Klls=19; IDls=12) were conducted, between April and May 2020. Three overarching themes emerged. The first was 'challenges faced by front-line healthcare providers working in COVID-19 wards. Healthcare workers experienced increased anxiety due to the fear of acquiring infection and transmitting it to their family members. They felt overwhelmed due to the exhaustive donning and doffing process, intense work and stigmatisation. The second theme was 'enablers supporting healthcare providers to deal with the COVID-19 pandemic'. Front liners pointed out several enabling factors that supported hospital staff including a safe hospital environment, adequate training, a strong system of information sharing and supportive management. The third theme was 'recommendations to support the healthcare workforce during the COVID-19 crisis'. Healthcare workers recommended measures to mitigate current challenges including providing risk allowance to front-line healthcare providers, preparing a backup health workforce, and establishing a platform to address the mental health needs of the healthcare providers.

Conclusion This study provides an initial evidence base of healthcare providers' experiences of managing patients with COVID-19 in the early stage of the pandemic and highlights measures needed to address the encountered challenges. It offers lessons for hospitals in low-income and middle-income countries to ensure a safe working environment for front-line workers in their fight against COVID-19.

\section{BACKGROUND}

Countries around the world are facing the unprecedented challenge and are struggling
Strengths and limitations of this study

- The front-line healthcare workers are uniquely positioned to address some of the most pressing issues related to the COVID-19 pandemic; thus, this study is positioned well to explore experiences of the barriers and facilitators to treat and manage COVID-19 cases.

- One limitation is that to minimise the risk of infection all study respondents were interviewed online over Zoom and hence the authors did not have the opportunity to build rapport with the respondents or obtain non-verbal cues during the interviews.

- The study was unable to conduct focus group interviews, due to the nature of outbreak prevention, which would have provided in-depth information about personal and group feelings.

to cope with the COVID-19 pandemic. ${ }^{1}$ First discovered in Wuhan, China; COVID-19 has swiftly travelled borders over the last couple of months leaving a trail of high morbidity and mortality with devastating effect on economies. $^{2}$ As of 22 June 2020, there have been 9071341 confirmed cases globally, with 471 162 deaths. $^{3}$ Moreover, 181088 COVID-19 cases and 3590 deaths have been reported in Pakistan. ${ }^{4}$

Pakistan shares borders with China and Iran; one being the epicentre of the disease and the other has seen an exponential increase of cases, respectively. ${ }^{5}$ The rapidly evolving pandemic has stressed the entire healthcare system of Pakistan and outpaced the capacity of hospitals to meet the demand for vital medical resources, such as ventilators, intensive care units beds, and personal protective equipment (PPE). ${ }^{5}$ The hospitals in Pakistan are in the midst of responding to the pandemic and are adopting urgent and innovative approaches. These include aspects such as: setting up designated isolation wards 
for patients diagnosed with COVID-19, procuring and distributing PPE, conducting screening and performing diagnostic tests, delaying non-emergency procedures and shifting from onsite to teleconsultation outpatient services. ${ }^{45}$

Since the time COVID-19 has hit countries, scientific evidence is clustering more around understanding the disease transmission and its pathogenicity. While disease epidemiology is important to understand the spread and risk factors, there is also a need to explore and understand experiences and perceptions of the health workforce involved in the COVID-19 crisis. $^{6}$ As the numbers are increasing, healthcare providers around the world are playing a central role and are making great contributions, while simultaneously facing great challenges. ${ }^{7}$ The frontline healthcare workers across the world are uniquely positioned to address some of the most pressing issues related to the COVID-19 pandemic such as: physical burn-out due to increase workload, mental exhaustion, fear of becoming infected and infecting others, sense of helplessness due to unavailability of personal protective gear, etc. ${ }^{8}$ Experiences from previous epidemics showed that while healthcare workers are often resilient, they require the same physical, psychological, and social support as others in times of turmoil. ${ }^{10-14}$ Initial research into the physical, emotional and psychological effects of COVID-19 on the health workforce managing COVID-19 patients in Wuhan showed that intensive work tends to drain front-line healthcare providers physically and emotionally. ${ }^{714}$

It is, therefore, significant to not only look to our current scientific knowledge but also to collect and interpret data on the specific ways this outbreak influences our front-line healthcare workers. This crisis situation necessitates investigating healthcare providers' perspectives and experiences of the barriers and facilitators to treat and manage COVID-19 cases. This study provides an understanding of the factors that are necessary to improve the experiences of healthcare providers dealing with the COVID-19 pandemic.

\section{METHODS}

\section{Study design and setting}

This formative research employed an exploratory qualitative research design using semi-structured interviews and a purposive sampling approach. The study was conducted at the private tertiary care teaching hospital in Karachi, Pakistan.

\section{Data collection methods and study participants}

The data collection methods for this formative research included key-informant interviews (KIIs) and in-depth interviews (IDIs). Key informants were purposively identified and recruited from senior management and hospital leadership, directly or indirectly involved with the management of COVID-19 patients. Similarly, participants for IDIs were also purposively recruited and included front-line healthcare providers, directly involved in the care of COVID-19 patients such as doctors, nurses, and pharmacists.

\section{Data collection procedure}

Semistructured interview guides were designed for KIIs and IDIs. The interview guides for KIIs and IDIs are provided in online supplemental annexes 1 and 2 The interview involved a discussion on perceptions about the COVID-19 outbreak, along with the barriers and facilitators experienced by healthcare providers while caring for COVID-19 patients. The interview guides were pilot tested with a non-study sample (two KIIs and two IDIs) with the same characteristics as the study sample. The pilot testing offered evidenced-base guidance to improve data collection guides. Before beginning the interview, the study investigators explained the study objectives and procedures to eligible healthcare providers and obtained informed consent for their participation in the study. Trained researchers, experienced in qualitative research, conducted online interviews using Zoom. The interviews were conducted in the languages of English and/or Urdu. Study participants were assured that their anonymity will be maintained. Informed consent was also obtained for notetaking and audio-recording of the interview.

\section{Key-informant interviews}

A total of 19 KIIs were conducted with senior management and hospital leadership. These KIIs were conducted to understand the barriers and facilitators faced while managing COVID-19 cases at Aga Khan University Hospital (AKUH). The key informants were electronically invited to participate in the qualitative study. Each KII took approximately between 30 and $45 \mathrm{~min}$.

\section{In-depth interviews}

IDIs were conducted with front-line healthcare providers including doctors, nurses and pharmacists who were directly involved in caring for COVID-19 patients. A total of 12 IDIs interviews were conducted with a group of front-line healthcare providers. The healthcare providers were identified from the AKUH COVID-19 inpatient wards and outpatient screening and testing areas. Frontline healthcare providers were electronically invited to participate in their off-duty hours. Each IDI took approximately between 30 and $45 \mathrm{~min}$.

\section{Data analysis}

Study data were analysed manually using the conventional content analysis technique. ${ }^{15}$ First, the audio recordings from the interviews were transcribed and then translated into the English language. No identifying characteristics were included in the transcriptions. Transcripts were read several times by four research investigators to develop an interpretation of the perspectives and experiences of the barriers and facilitators to treat and manage COVID-19 cases. This involved an iterative process where data were coded, compared, contrasted and refined to generate emergent themes. The transcribed text was divided into 
'meaning units' which were later shortened and labelled with a 'code' without losing the study context. Codes were then analysed and grouped into similar categories. In the final step, similar categories were assembled under subthemes and main themes. Two independent investigators (NAP and ASF) performed the coding, and category creation and discrepancies were resolved through discussion until a consensus was reached.

\section{Trustworthiness of the study}

Tracy et $a l^{16}$ and Lincoln and Guba's criteria ${ }^{17}$ were used to establishing trustworthiness and methodological rigour. To ensure credibility, the study triangulated data via two basic types of triangulation: data source triangulation (exploring insights of different groups-healthcare providers and key-informants) and investigator triangulation (use of multiple researchers in analysis phase-NAP and ASF).$^{18}$ Study rigour was also be enhanced through member checking of transcripts and synthesised data to confirm whether study results have resonance with the participants' experience. ${ }^{19}$ Since our study used a qualitative approach, it was more interested in gaining an understanding of providers' experiences of COVID-19 management rather than aiming at singular truth and generalisation. Patton and Rolfe emphasise that the qualitative inquiry often prioritise depth over breadth through studying smaller samples and even single case and often makes very limited claims about the study external validity. ${ }^{201}$

\section{Patient and public involvement}

Patient public involvement is a relatively new concept in Pakistan. Our data collection tool was piloted through two IDIs to ensure that it is inclusive and comprehensive. Front-line healthcare providers were not involved in the development of research questions and design, and data collection decisions.

\section{RESULTS}

In this qualitative study, 19 KIIs and 12 IDIs were conducted, between April and May 2020, with a variety of participants including, residents, registered nurses, head nurses, nurse managers, pharmacists, senior management and few key individuals from leadership positions. Data collection was ceased once saturation was achieved; saturation refers to the point in the research process when no new information is discovered in data analysis ${ }^{22}$ The small number of qualitative interviews allowed us to dug into the depth of each interview to understand the unique perspectives and experiences of healthcare providers regarding COVID-19. The demographic information for the KIIs and IDIs participants is illustrated in table 1. All the study participants $(n=31)$ who were approached by the study team agreed to participate in the study.

Based on the data collection and thematic analysis, three overarching themes were identified (1) Challenges faced by front-line healthcare providers working
Table 1 Characteristics of KIls and IDI study participants $(\mathrm{KII}=19 ; \mathrm{IDI}=12)$

\begin{tabular}{|c|c|}
\hline & $\mathbf{N}(\%)$ or median (range) \\
\hline \multicolumn{2}{|c|}{ Characteristics of KII participants } \\
\hline \multicolumn{2}{|l|}{ Gender } \\
\hline Female & $11(57.9 \%)$ \\
\hline Male & $8(42.1 \%)$ \\
\hline Age & $45(34-58)$ \\
\hline \multicolumn{2}{|l|}{ Designation } \\
\hline Professor & $5(26.3 \%)$ \\
\hline Associate professor & 7 (36.8\%) \\
\hline Assistant professor & $2(10.5 \%)$ \\
\hline Manager & $3(15.8 \%)$ \\
\hline Leadership role & $2(10.5 \%)$ \\
\hline Years of experience & $19(1-45)$ \\
\hline \multicolumn{2}{|c|}{ Characteristics of IDI participants } \\
\hline \multicolumn{2}{|l|}{ Gender } \\
\hline Female & $11(91.7 \%)$ \\
\hline Male & $1(8.3 \%)$ \\
\hline Age & $31(22-48)$ \\
\hline \multicolumn{2}{|l|}{ Designation } \\
\hline Doctor & $5(41.7 \%)$ \\
\hline Nurse & $6(50 \%)$ \\
\hline Pharmacists & $1(8.3 \%)$ \\
\hline Years of experience & $9(2-20)$ \\
\hline
\end{tabular}

IDI, in-depth interview; KII, key-informant interview.

in COVID-19 wards; (2) Enablers supporting healthcare providers to deal with COVID-19 pandemic and (3) Recommendations to support healthcare workforce during the COVID-19 crisis. The themes and categories are presented in table 2.

Themes 1: challenges faced by front-line healthcare providers working in COVID-19 wards

Concerns about the management of COVID-19 cases

While front-line healthcare providers and senior management expressed their determination to offer services in these challenging times to manage COVID-19 patients, various concerns related to the treatment and management of COVID-19 cases were articulated. Dialogues with hospital senior management representatives indicated that standard operating procedures (SOPs) have been designed to manage COVID-19 cases, however, few frontline care providers believed that the presence of SOPs is fairly ambiguous. Expressing similar concerns, a pharmacist stated:

I have concerns about how to deal with patients ... We need a clear procedure for dealing with them. For example, when someone comes into the pharmacy, what procedures are we meant to follow (IDI-08, Pharmacist). 


\begin{tabular}{ll}
\hline Table 2 Themes and categories & Categories \\
\hline Themes & Concerns about the management of COVID-19 cases \\
\hline $\begin{array}{l}\text { Challenges faced by front-line healthcare providers } \\
\text { working in COVID-19 wards }\end{array}$ & Overwhelmed and exhausted by the workload and exhaustive donning and doffing \\
& Thecess \\
Enablers supporting healthcare providers to deal with & Adequate training and drills for dealing with COVID-19 cases \\
COVID-19 pandemic & The strong hospital system of information sharing during the COVID-19 crisis \\
& Supportive management and leadership \\
Recommendations to support healthcare workforce & Ensuring motivation for front-line health workforce \\
during the COVID-19 crisis & Anticipate and address the mental health needs of the health workforce \\
&
\end{tabular}

During interviews, an insight into the initial practices of managing the COVID-19 crisis was also probed. Senior hospital management mentioned that the hospital was fully prepared to manage this public health emergency since its epidemic in China. Contrary to this, front-line physicians and nurses verbalised glimpses of an ad hoc management of outpatient hospital areas for COVID-19 screening and testing, during the early phase of the pandemic. Furthermore, on one hand, senior management generally exhibited their satisfaction over the availability and provision of PPEs to front-line healthcare providers. While on the other hand, shortages of PPEs were notified by few healthcare providers, alongside sanitisers while providing care to the patients.

Sometimes we face a shortage of sanitizers and other essential PPEs such as masks. I think that all the PPEs should be available at all times so that we are not worried. Sometimes doctors ask us to bring them an N95 mask and we are unable to do so because we do not have any (IDI-02, Nurse)

Although respondents appreciated the availability of negative pressure rooms during the COVID-19 pandemic, concerns about the limited capacity of the hospital were verbalised for the efficient and timely management of COVID-19 cases. Few hospital staff reported that they experienced violent behaviour by the family in case of refusal to admit new patients.

Fear of acquiring infection and transmitting to family members Due to the highly contagious nature of the coronavirus (SARS-CoV-2) and perceived uncertainty in contracting the disease, interviews with physicians and nurses revealed their apprehension in acquiring the virus while treating patients. The front-line workers face a unique mental health challenge and several respondents experienced feeling guilty about potentially carrying the virus to their families. Highlighting this point, one respondent stated:

It is a stressful situation. By the end of the day when I am taking a break, I have many negative thoughts. I worry about carrying this infection to my family...I have a young daughter at home and nearly every day
I worry about being asymptomatic and carrying this infection to my family (IDI-07, Doctor).

The increased likelihood of contracting COVID-19 is also psychologically affecting the senior management team across the hospital. Due to their exposure; their family members are also at risk of acquiring the infection.

I work in the emergency department so I always have a fear that the next patient I see will need serious treatment and I may have to resuscitate him/her. But now, I always have a fear that the next patient will be Covid-19 positive and that they may infect me. And if I get infected my family will get infected. So, this fear is a little bit different and it will last till the pandemic last (IDI-12, Doctor)

While verbalising the concerns about the healthcare providers' exposure in getting infected, respondents also voiced their concerns that front line staff is at high risk of getting infected even in non-COVID-19 areas across the hospital setting.

\section{Overwhelmed and exhausted by the workload and exhaustive} donning and doffing process

To protect front-line workers against COVID-19, the infection control policy at AKUH mandates that all staff working in areas where COVID-19 patients are suspected wear a full sleeve impervious gown, gloves, and an N95 mask. While this policy is no doubt effective and in line with the best interest of the front-line workers, it poses several challenges. For instance, our interviews revealed that several participants found the N95 mask suffocating to wear for a prolonged period of time. Commenting on the experience of wearing full PPE one front-line worker stated:

We...get tired of wearing full PPE because we have to be in the room with the patient for four hours. It gets really hot and the extra layers of protection weigh heavy on the body (IDI-11, Nurse).

Moreover, another respondent highlighted how the process of using PPE is complicated when staff is required to visit one patient to another. This occurs because 
the staff has to meticulously switch in and out of PPEs. Therefore, what was initially a mundane process has now become a critical aspect of infection control. This point was illustrated by a respondent who stated:

It takes around 5-7 min to put on our PPEs. We then go to the patient's room... come back and spend the same amount of time to switch our PPEs before going to the next patient's room. This process is a big hassle and is time-consuming. But we have to be extra careful, if this procedure is not done properly, we can pass on the infection (IDI-07, Doctor).

While this process is no doubt challenging, one respondent offered an encouraging remark stating:

Initially we felt that our workload has increased, however, with the passage of time we have become used to it and things feel normal (IDI-16, Nurse).

\section{The stigma associated with healthcare providers working in}

\section{COVID-19 wards}

COVID-19 is primarily transmitted from symptomatic people to others through direct contact, or by contact with contaminated objects and surfaces. Moreover, a large portion of those infected is asymptomatic, meaning they show no overt markers of the infection. As a result of this, front-line workers face a unique mental health challenge. Since they work in high-risk environments many opt to hide details about their work-life in fear of being stigmatised by their communities.

I know that in some cases health care workers do not tell their families and communities that they are working with Covid-19 patients. They fear that this will cause unnecessary panic and people may view them differently (KII-19, Associate Professor).

It is likely that this anxiety within the families and communities of healthcare workers is propagated by the ambiguity of information available on COVID-19. It is possible that the hospital may address many of these issues by extending its outreach services. During the interviews, a front-line worker was critical of the hospital's current outreach services:

Our services should be extended to the community. Compared to other institutions we have not done enough. Many people have criticized us in this pandemic (IDI-08, Pharmacist).

By providing more extensive services to surrounding communities, the hospital could not only alleviate the stigma faced by front-line workers but also reduce the surge of false information.

\section{Theme 2: enablers supporting healthcare providers to deal with COVID-19 pandemic}

A safe and secured hospital environment

Many respondents stated that the hospital has provided a safe environment for employees and that safety measures have been improved as the hospital administration became more knowledgeable about the nature of this disease. One respondent stated the hospital's disaster management and incident command system were ensuring adequate training and smooth communication throughout the hospital.

When the number of cases started increasing, the hospital enacted the Hospital Incident Command System, leaders from each of the different areas that is, logistics, communications, medicine, etc came together to make sure that everything was in place-. The hospital has now made smaller groups which meet regularly to go over each of the issues and an executive Operations Command Committee goes over what should be done. (KII-19, Associate Professor)

While provisions are made for the availability of PPE for the staff, many noted that implementation of proper usage of PPE and adequate hand hygiene is still a problem that requires behavioural change. It was also stated that healthcare providers that are considered vulnerable (ie, elderly and/or have serious pre-existing conditions) are not allowed to work in the COVID-19 established areas.

While several precautions are being taken, one respondent claimed that there were many places for improvement. One stated that it is complacent to feel good about any sense of safety and security and that it is important to remain vigilant in the case of new information about the disease or a high influx of patients.

There are several places where things can slip through the cracks, and cause problems, and there are several points that will fail if they come under pressure- I am not absolutely confident, but it is good so far. (KII- 16, Professor)

\section{Adequate training and drills for dealing with COVID-19 cases}

When questioned about training and drills, most respondents stated that everyone who is working for COVID-19 is trained in the usage of PPE, N95 mask, donning and doffing, and taking test samples using nasopharyngeal swabs. Many also said that regular training was being carried out on the job and at the CIME, and that master trainers were being trained to then disseminate information and train the rest of the department. Covinars (COVID-19 Webinar) sessions are being conducted to help train and provide information about the disease.

We have completed 2-day training workshops or seminars and get trained every day in new technologies and when new guidelines come (IDI-02, Nurse)

However, some specialists were concerned that very little had been done in the way of training; it was noted that besides guidance on the N95 mask fitting test, there were no opportunities to go through any drills. While communications were being carried out, it was not considered sufficient. Others said that while training were being conducted, they were not very regular. While they stated 
that this may be because of the social distancing measures, they insisted on more regular online training.

The strong hospital system of information sharing during the COVID-19 crisis

While the outbreak of COVID-19 has put immense pressure and stress on the hospital staff, many facilitators support the hospital staff to deal with the pandemic. Respondents stated that information was being shared through video messages and that helplines and hotlines for staff and the public were effective in screening for COVID-19 symptoms. While many stated that information sharing was difficult at first, it was claimed that this was due to the changing information coming about the disease from international agencies It was reported that the hospital leadership holds weekly meetings with senior management, who then circulate that within their respective departments.

I think we have a reasonably good system built for disaster and we have a very defined chair of command... There have been different working groups formed for Covid-19 and they all have specialized tasks for information sharing, and there is a Covid-19 hotline for employees and the public and that is adequate. (KII-16, Professor)

In addition, university-wide town hall meetings were held regularly. It was suggested that more town halls should be carried out and that regular memos should be sent with information about caring for those with the disease.

Town halls boosted the morale of the health care providers, and this communication was very good. (KII-3, Professor)

\section{Supportive management and leadership}

Most IDI participants mentioned that senior management and institutional leadership is providing immense support by ensuring appropriate PPE in the COVID-19 and non-COVID-19 wards to ensure the safety of frontline healthcare providers. In addition, few participants mentioned that the institutional leadership regularly visits COVID-19 units for staff appreciation and encouragement. Besides, the senior management responds to healthcare providers' concerns in a timely manner through a WhatsApp group.

Initially, we were supposed to remain inside the patient room consecutively for 4 hours. This was very exhausting for bedside nurses especially since we have to wear three layers of PPE. We raised these concerns and senior management has now permitted us to exit the room when the patient's condition gets stable ... we now observe the patients from the mirrored door. This has given us a huge relief (IDI-11, Nurse)

While front-line providers appreciated the support received from management and institutional leadership, they also recognised the efforts of all other support departments who are working together for the safety of front-line hospital staff. These support departments include finance, design office, construction, laundry, purchase and supply chain management, safety and security, human resource, information, and technology department, nutrition and food services, marketing and communications, travel services, etc.

All the support departments are contributing in the same manner as our frontline healthcare providers (KII-01, Professor)

In addition, the few IDI participants mentioned that institutional leadership has arranged accommodation facilities for the front-line staff who are working in COVID-19 wards but the hospital staff is not availing those services because they have their families and children back home. Few respondents further stated that the senior management has also ensured the provision of shower facilities for the front-line hospital staff; however, due to time limitations, nurses are unable to make effective use of those facilities.

\section{Theme 3: recommendations to support the healthcare workforce during the COVID-19 crisis \\ Prepare and train backup health workforce}

Both IDI and KII participants mentioned that they have been experiencing staff shortages in COVID-19 wards because many of the front-line healthcare providers have been either quarantined or isolated due to exposure. When asked about recommendations to support the front-line health workforce, most IDI respondents suggested that healthcare providers (doctors and nurses) of other sub-specialties (neurology, cardiac, surgery, orthopaedic) need to be trained as a backup to mitigate situations when entire internal medicine teams may be placed in self-quarantine due to COVID-19 exposure. In addition, a few key informants recommended that there should be a central backup plan for staff coverage in both COVID-19 and routine wards.

Currently, only the healthcare providers of Covid-19 wards have received specialized training on ventilator code, BIPAP management, and handling body of expired Covid-19 patient. However, these training should be given to all healthcare staff across the institution to prepare a central backup. (IDI-03, Nurse)

\section{Ensuring motivation for front-line health workforce}

To ensure enthusiasm among front liners, study respondents highlighted the need of appreciating and motivating front-line providers for their countless efforts in this pandemic battle.

Most IDIs including front-line nurses suggested that risk allowance should be given to all front-line healthcare providers involved in the treatment and management of COVID-19 patients. Healthcare providers suggested that instead of giving extra time off, hospital staff should be 
compensated for taking additional risks while caring for COVID-19 cases.

As you know the world is very materialistic and people always need motivation. While we are being encouraged by senior management, this form of verbal motivation will only work for a time period. If the current situation is going to go on, we will need to give people an added incentive in the form of material compensation. This can either be more money or additional days off (IDI-04, Nurse).

In addition, respondents verbalised that some activities for staff entertainment should also be thought-about to alleviate stress and anxiety associated with this crisis situation among healthcare providers.

\section{Anticipate and address the mental health needs of the health} workforce

IDI and KII respondents mentioned that there is no formal platform established where front liners' voices are being heard. Such a platform could provide an opportunity to anticipate and address the mental health needs of the front-line health workforce.

Everyone is very stressed. I see it every time one of my staff has to take care of a suspected patient, they are hesitant and scared. Sometimes, I feel the same way myself. We need an integrated counseling program. People should not just come for counseling when they are mentally struggling. Similar to how we have guidelines for PPE and social distancing we should have small group talks on ZOOM so that we can dispel our anxieties before they build up (KII 06, Associate Professor).

Few study participants particularly KIs appreciated the motivation sessions organised by psychiatric fellows on stress and coping. However, study respondents highlighted the need of arranging more psychiatric sessions for healthcare staff on a daily basis to cope with the stress. More specifically, participants stated that currently there is a blanket approach around mental health; however, more is needed to address varied concerns of the health workforce.

There was a zoom session arranged on stress management, but I was unable to attend it due to my duties. I think we need more of these sessions. We can even add more innovative things such as breathing exercises, mindfulness, and yoga. There is so much anxiety relating to Covid-19 both at work and in our homes. Everyone is so panicked and there is so much hype going around. These types of innovative sessions would really help (IDI-05, Doctor)

\section{DISCUSSION}

To the best of our knowledge, this is the first study to explore perceptions and experiences of healthcare providers during the COVID-19 pandemic in Pakistan. The research identified challenges faced by healthcare providers while managing COVID-19 patients, alongside strategies to cope with these. The front-line healthcare providers pointed out several concerns that influenced their ability and willingness to treat and manage COVID-19 patients. These included shortage of PPEs and hand sanitisers, lack of clear SOPs, ad hoc management of hospital outpatient area for COVID-19 screening and testing, violent behaviour by families of COVID-19 positive patient and limited capacity of the hospital to treat and manage increasing COVID-19 positive patients. Notwithstanding some challenges that cannot be mitigated by the institution such as the increasing number of COVID-19 positive patients and unexpected violent behaviour of families of COVID-19 positive patients, a number of corrective actions that can be taken to lessen the impact of others.

Our results highlight, especially in the initial period of the crisis, differences in the responses received from senior management and front-line providers with regard to the availability of PPEs and sanitisers, presence of clear SOPs, preparedness of hospital to manage COVID-19 pandemic. These discrepancies could be partly due to the communication gap between the two group of respondents (whereby senior management and hospital leadership was heavily involved in the process of designing new screening and testing site, procuring PPE and updating SOPs considering the differential progression of the outbreak). Most have now been addressed through corrective actions by the hospital leadership during the last few weeks and months. As this is a leading private teaching hospital in the country, the senior management and hospital leadership was able to successfully address the gaps to improve the experiences of front liners involved in this pandemic. However, this may not be the case in most public sector hospitals, where frontline healthcare workers continue to face challenges. The healthcare systems in low-income and middle-income countries face serious constraints in capacity and accessibility during normal times. This would be aggravated during the COVID-19 outbreak, leading to worse clinical outcomes, poor-quality healthcare and poor healthcare workers' experiences. ${ }^{23} 24$

Consistently with experiences from previous outbreaks and emergencies, ${ }^{2325}$ front-line healthcare workers providing care to COVID-19 patients experienced increased anxiety and stress. Our study found that the increased exhaustion among healthcare providers is due to the fear of acquiring infection and transmitting it to their family members. Anxiety and burnout among healthcare providers were also reported by studies conducted in high-income countries although countries were combating different stages of the pandemic. ${ }^{23}$ This may be due to standard changes in working hours, shortage in a skilled workforce and inadequate access to PPE. ${ }^{23}$ The front-line providers in our study felt overwhelmed due to the exhaustive donning and doffing process, intense work, and a large number of patients, which was consistent with the studies on the outbreak of MERS-Cov ${ }^{26} 27$ and Ebola. ${ }^{28}$ 
Our results undoubtedly show that stigma is a pressing issue for the front-line healthcare workers working in COVID-19 wards. Several studies have reported that there are several potential mechanisms by which stigma could affect healthcare providers' outcomes, ${ }^{29}{ }^{30}$ and HCPs who experience higher levels of stigma reported increased physical (fatigue) and psychological distress (burn-out). ${ }^{31}$ These pressures can lead to mental health problems, for example, burn-out, anxiety, depression, insomnia, denial, anger, which not only influence front-line healthcare providers' attention, understanding and decision-making capacity but could also have a long-lasting impact on their physical and psychological health after the COVID-19 emergency is over. ${ }^{7}$

While the outbreak of COVID-19 put immense pressure and stress on the hospital staff, many enabling factors supported hospital staff to deal with these aspects, which have progressively evolved over the duration of the pandemic. As a result of this pandemic, the entire hospital was able to pull together and many departments across the university hospital coordinated to ensure smooth and efficient operations. Findings suggest that the respondents felt that they were actively encouraged and supported by senior management and the university leadership. More specifically, the research subjects felt that over time the safe and secured hospital environment enabled healthcare providers to perform their routine tasks and reduce their feeling of uncertainty and fear. Similar findings have been reported by the qualitative study published in Lancet Global Health by Liu et al. ${ }^{32}$ Our study found that the healthcare providers were appreciative of the training provided to them regarding the use of PPE, N95 mask, donning and doffing, and taking test samples using nasopharyngeal swabs. However, it was reported that more drills could be conducted to improve their hands-on skills and reduce the risk of acquiring infection. Health workforce safety is a high priority and therefore it is essentially important to provide sufficient protective supplies and training and drills for effective management of COVID-19 cases. ${ }^{32} \mathrm{~A}$ unique yet encouraging finding reported by our study participants was that the hospital developed a strong system of information sharing to keep faculty and staff updated about the COVID-19 situation through video messages, hotlines, town halls and what are now called Covinars.

Our study also reported some recommendations to mitigate current challenges and further improve the experiences of healthcare providers working in COVID-19 wards. The front-line providers caring for COVID-19 patients felt extreme physical discomfort and fatigue due to long working hours and complicated donning and doffing process and suggested that institutions should provide risk allowance to compensate healthcare providers for the additional risks they take and to motivate staff to continue to work. This finding is consistent with the previous experience from the outbreak of Ebola in western Africa, where risk allowance was adopted as a strategy for motivating and retaining healthcare workers. ${ }^{33}$ Our study suggested preparing and cross-train a backup health workforce to effectively respond to staff shortages as many of the frontline healthcare providers have been either quarantined or isolated due to exposure. Similar recommendations have been provided by a number of studies conducted in diverse settings. ${ }^{34-36}$ Our study also showed that a formal platform where front liners' voices could be heard did not exist. Respondents reported that such a platform could provide an opportunity to anticipate and address the mental health needs of the front-line health workforce. Experiences from similar outbreaks suggest that early psychological intervention and establishment of early support systems are particularly important for front liners to promote the emotional release and improve healthcare providers' mental health. ${ }^{37}$

This study was conducted in a leading private tertiary care teaching hospital in Karachi, Pakistan that offers state-of-the-art healthcare. The initial challenges progressively led to a fairly successful story. The same cannot be said for a large number of public and private hospitals in the country. The shortage of PPE has been a frequent occurrence and has even led to public protests, undoubtedly contributing to mental stress and distress. The experience gained from the current study offers lessons for other hospitals in the country to benefit from. There is no doubt that good quality healthcare against COVID-19 can only be ensured if the front-line workers are well taken care of in terms of their mental health and physical needs when asked to serve critically ill patients round the clock.

This study provides an initial evidence base of healthcare providers' experiences of managing COVID-19 patients in an early stage of the pandemic when the participants just accepted the antiepidemic tasks. Diverging from the findings of various studies on the experience of negative emotions and barriers encountered during the outbreak, we found that facilitators coexist with challenges, which supported front liners to effectively deal with the crisis. The findings from this study can be directly used for improving preparedness and response for possible future COVID-19 waves or other outbreaks. Future research could be conducted to perform an in-depth analysis of before-and-after pandemic conditions and their influence on healthcare providers' experiences.

\section{Methodological limitations}

The study sample was small, particularly when considering the AKUH employs over 6300 healthcare providers, yet our study intent was to be dug into the depth of each interview to understand unique perspectives and experiences of healthcare providers regarding COVID-19. Bengtsson et al suggest that the qualitative researcher has often to choose depth over breadth to gain a rich understanding of a phenomenon. ${ }^{38}$ However, there remains a possibility that our sample belongs to a particular subgroup of healthcare providers who were motivated to engage with the study to inform a particular story for their COVID-19 experience. Another limitation of this study was that all study respondents were interviewed online, to minimise the risk of infection. The authors did not have the opportunity to build rapport with respondents over Zoom or obtain non-verbal cues during interviews. Due to the nature of outbreak prevention, our study was unable to 
conduct focus group interviews, which would have provided detailed information about personal and group feelings. Lastly, this was a short-term study and does not include longterm experiences of the research subjects with this pandemic.

\section{CONCLUSION}

This study provides an in-depth understanding of the healthcare providers' experiences of the COVID-19 outbreak and emphasises that adequate training and drills, sufficient PPE, a safe and secured hospital environment, healthcare providers motivation, supportive hospital management and leadership, strong hospital system of information sharing and psychological support to address mental health needs of front liners are necessary to improve the overall experiences of healthcare providers fighting COVID-19.

Acknowledgements The authors would like to acknowledge Dean Adil Haider of Medical College, Aga Khan University Karachi, Pakistan for his greater involvement in conceiving the study concept.

Contributors All authors had full access to all the data in this study and take responsibility for the integrity of the data and the accuracy of the data analysis. SSa, SSi, ASF, NAP, ZHA and NA designed the study. ASF supervised data collection and analysis. ASF, NAP, ZHA and MMS collected the data. ASF, NAP, ZHA, MMS, SSa analysed and interpreted the data. ASF, NAP, ZHA and MMS wrote the first draft of the manuscript. All authors contributed to reviewing and editing the manuscript.

Funding The authors have not declared a specific grant for this research from any funding agency in the public, commercial or not-for-profit sectors.

Competing interests None declared.

Patient consent for publication Not required.

Ethics approval This study was obtained from the Aga Khan University Ethical Review Committee (AKU-ERC) - (2020-3694-9056). Written informed consent was provided by all study participants. Informed consent included permission to audio record the interviews and use anonymised quotes. Voluntary participation and the right to ask any questions and to decline participation at any time were emphasised during the data collection.

Provenance and peer review Not commissioned; externally peer reviewed.

Data availability statement Data are available on reasonable request. The datasets used and/or analysed during the current study are available from the corresponding author on reasonable request.

Supplemental material This content has been supplied by the author(s). It has not been vetted by BMJ Publishing Group Limited (BMJ) and may not have been peer-reviewed. Any opinions or recommendations discussed are solely those of the author(s) and are not endorsed by BMJ. BMJ disclaims all liability and responsibility arising from any reliance placed on the content. Where the content includes any translated material, BMJ does not warrant the accuracy and reliability of the translations (including but not limited to local regulations, clinical guidelines, terminology, drug names and drug dosages), and is not responsible for any error and/or omissions arising from translation and adaptation or otherwise.

Open access This is an open access article distributed in accordance with the Creative Commons Attribution Non Commercial (CC BY-NC 4.0) license, which permits others to distribute, remix, adapt, build upon this work non-commercially, and license their derivative works on different terms, provided the original work is properly cited, appropriate credit is given, any changes made indicated, and the use is non-commercial. See: http://creativecommons.org/licenses/by-nc/4.0/.

\section{ORCID iDs}

Anam Shahil Feroz http://orcid.org/0000-0003-0180-0213

Sameen Siddiqi http://orcid.org/0000-0001-8289-0964
REFERENCES

1 World Health Organization. Rolling updates on Covid.19, 2020. Available: https://www.who.int/emergencies/diseases/novelcoronavirus-2019/events-as-they-happen

2 Tian H, Liu Y, Li Y, et al. An investigation of transmission control measures during the first 50 days of the COVID-19 epidemic in China. Science 2020;368:638-42.

3 Corrigan P. On the Stigma of COVID-19. Let's separate the illness from the patient, 2020. Available: https://www.psychologytoday.com/ us/blog/the-stigma-effect/202004/the-stigma-covid-19

4 Saqlain M, Munir MM, Ahmed A, et al. Is Pakistan prepared to tackle the coronavirus epidemic? Drugs Ther Perspect 2020:213-4.

5 Waris A, Atta UK, Ali M, et al. COVID-19 outbreak: current scenario of Pakistan. New Microbes New Infect 2020;35:100681.

6 Mahar I. Pakistan: Covid-19: rhetoric and reality, 2020. Available: http://southasiajournal.net/pakistan-covid-19-rhetoric-and-reality/

7 Xiong Y, Peng L. Focusing on health-care providers' experiences in the COVID-19 crisis. Lancet Glob Health 2020;8): :e740-1.

8 Sim MR. The COVID-19 pandemic: major risks to healthcare and other workers on the front line. Occup Environ Med 2020;77:281-2.

9 Chew NWS, Lee GKH, Tan BYQ, et al. A multinational, multicentre study on the psychological outcomes and associated physical symptoms amongst healthcare workers during COVID-19 outbreak. Brain Behav Immun 2020;88:559-65.

10 Teti M, Schatz E, Liebenberg L. Methods in the time of covid-19: the vital role of qualitative inquiries. Los Angeles, CA: SAGE Publications Sage CA, 2020.

11 Faulkner SL, Trotter SP. Data saturation. IECRM 2017:1-2.

12 Hsieh H-F, Shannon SE. Three approaches to qualitative content analysis. Qual Health Res 2005;15:1277-88.

13 Abdelhafiz AS, Mohammed Z, Ibrahim ME, et al. Knowledge, perceptions, and attitude of egyptians towards the novel coronavirus disease (COVID-19). J Community Health 2020;45:881-90.

14 Chang $\mathrm{D}$, Xu H, Rebaza A, et al. Protecting health-care workers from subclinical coronavirus infection. Lancet Respir Med 2020;8:e13.

15 Li L-Q, Huang T, Wang Y-Q, et al. COVID-19 patients' clinical characteristics, discharge rate, and fatality rate of meta-analysis. $J$ Med Virol 2020;92): :577-83.

16 Tracy SJ. Qualitative quality: eight "big-tent" criteria for excellent qualitative research. Qualitative Inquiry 2010;16:837-51.

17 Lincoln YS, Guba EG. Naturalistic inquiry. 75. CA: Sage Thousand Oaks, 1985.

18 Hussain T, Smith P, Yee LM. Mobile phone-based behavioral interventions in pregnancy to promote maternal and fetal health in high-income countries: systematic review. JMIR Mhealth Uhealth 2020;8:e15111.

19 Henry P. Rigor in qualitative research: promoting quality in social science research. Res J Recent Sci 2015;4:25-8 http://www.isca.in/ rjrs/archive/v4/ilVC-2015/6.ISCA-IVC-2015-20SHS-11.php

20 Patton MQ. Qualitative evaluation methods, 1980. https://www. gwern.net/docs/sociology/1980-patton-qualitativeevaluatio nmethods.pdf

21 Rolfe G. Validity, trustworthiness and rigour: quality and the idea of qualitative research. J Adv Nurs 2006;53:304-10.

22 Saunders B, Sim J, Kingstone T, et al. Saturation in qualitative research: exploring its conceptualization and operationalization. Qual Quant 2018;52:1893-907.

23 Semaan A, Audet C, Huysmans E, et al. Voices from the frontline: findings from a thematic analysis of a rapid online global survey of maternal and newborn health professionals facing the COVID-19 pandemic. BMJ Glob Health 2020;5:e002967.

24 Hopman J, Allegranzi B, Mehtar S. Managing COVID-19 in low- and middle-income countries. JAMA 2020;323:1549-50.

25 McMahon SA, Ho LS, Brown $\mathrm{H}$, et al. Healthcare providers on the frontlines: a qualitative investigation of the social and emotional impact of delivering health services during Sierra Leone's Ebola epidemic. Health Policy Plan 2016;31): :1232-9.

26 Kim Y. Nurses' experiences of care for patients with Middle East respiratory syndrome-coronavirus in South Korea. Am J Infect Control 2018;46:781-7.

27 Kang HS, Son YD, Chae S-M, et al. Working experiences of nurses during the Middle East respiratory syndrome outbreak. Int J Nurs Pract 2018;24:e12664.

28 Kraft CS, Mehta AK, Varkey JB, et al. Serosurvey on healthcare personnel caring for patients with Ebola virus disease and Lassa virus in the United States. Infect Control Hosp Epidemiol 2020;41:385-90.

29 Markowitz FE. The effects of stigma on the psychological well-being and life satisfaction of persons with mental illness. $J$ Health Soc Behav 1998;39:335-47. 
30 The Lancet. The health crisis of mental health stigma. Lancet 2016;387:1027.

31 Ramaci T, Barattucci M, Ledda C, et al. Social stigma during COVID-19 and its impact on HCWs outcomes. Sustainability 2020;12:3834.

32 Liu Q, Luo D, Haase JE, et al. The experiences of health-care providers during the COVID-19 crisis in China: a qualitative study. Lancet Glob Health 2020;8:e790-8.

33 Chersich MF, Gray G, Fairlie L, et al. COVID-19 in Africa: care and protection for frontline healthcare workers. Global Health 2020;16:1-6.
34 Dewey C, Hingle S, Goelz E, et al. Supporting clinicians during the COVID-19 pandemic. Ann Intern Med 2020;172:752-3.

35 Hasan Z, Narasimhan M. Preparing for the COVID-19 pandemic: our experience in New York. Chest 2020;157:1420-2.

36 Adams JG, Walls RM. Supporting the health care workforce during the COVID-19 global epidemic. JAMA 2020;323:1439-40.

37 Sun N, Wei L, Shi S, et al. A qualitative study on the psychological experience of caregivers of COVID-19 patients. Am J Infect Control 2020;48:592-8.

38 Bengtsson M. How to plan and perform a qualitative study using content analysis. NursingPlus Open 2016;2:8-14. 